\title{
Analysis of the Sales Checkout Operation in Supermarket Using Queuing Theory
}

\author{
Priyangika J.S.K.C ${ }^{1, *}$, Cooray T.M.J.A ${ }^{2}$ \\ ${ }^{1}$ Department of Physical Sciences, Faculty of Applied Sciences, Rajarata University of Sri Lanka, Sri Lanka \\ ${ }^{2}$ Department of Mathematics, Faculty of Engineering, University of Moratuwa, Sri Lanka
}

Copyright $\bigcirc 2016$ by authors, all rights reserved. Authors agree that this article remains permanently open access under the terms of the Creative Commons Attribution License 4.0 International License

\begin{abstract}
Waiting lines or queues are a common phenomenon in life, especially in the province of organizations that are for profit making. Queues are common in such places as petrol or filling stations, supermarkets stores, clinics, hospitals, motor parks, manufacturing firms, to mention a but a few. An interesting aspect of queuing process resides in the measures of its system's performance, especially in terms of average service rate, systems, utilization and the costs implied for a given capacity level. This paper explains the analysis of Queuing systems for the empirical data of supermarket checkout service unit using queuing theory. One of the expected gains from studying queuing systems is to review the efficiency of the models in terms of utilization and waiting length, hence increasing the number of queues so customers will not have to wait longer when servers are too busy. In other words, trying to estimate the waiting time and length of queue(s), is the aim of this study. Queuing simulation is used to obtain a sample performance result and estimated solutions for multiple queuing models are also interested. This study requires an empirical data which may include the variables like, arrival time in the queue of checkout operating unit (server), departure time, service time, etc. A questionnaire is developed to collect the data for such variables and the reaction of the Supermarket from the customers separately. This model is developed for a sales checkout operation in the supermarket. The model designed for this research is multiple queues multiple-server model. The model contains five servers which are checkout sales counters; attached to each server is a queue. In any service system, a queue forms whenever current demand exceeds the existing capacity to serve. This occurs when the checkout operation unit is too busy to serve the arriving costumers, immediately.
\end{abstract}

Keywords Multiple-server Model, Queuing Simulation, Service Time, Inter-arrival Time

\section{Introduction}

Delays and queuing problems are most common features not only in our daily-life situations such as at a bank or postal office, at a ticketing office, in public transportation or in a traffic jam but also in more technical environments, such as in manufacturing, computer networking and telecommunications. They play an essential role for business process re-engineering purposes in administrative tasks. "Queuing models provide the analyst with a powerful tool for designing and evaluating the performance of queuing systems." (Bank, Carson, Nelson \& Nicol, 2001).

Whenever customers arrive at a service facility, some of them have to wait before they receive the desired service. It means that the customer has to wait for his/her turn, may be in a line. Customers arrive at a service facility with several queues, each with one server (sales checkout counter). The customers choose a queue of a server according to some mechanism (e.g., shortest queue or shortest workload). (Adan, 2000).

This paper is the review of queuing theory and for empirical study the sales checkout service unit of a supermarket is chosen as an example.

The main purpose of this paper is to review the application of queuing theory and to evaluate the parameters involved in the service unit for the sales checkout operation in the Supermarket. Therefore, a mathematical model is developed to analyze the performance of the checking out service unit. Two important results need to be known from the data collected in the supermarket by the mathematical model: one is the 'service rate' provided to the customers during the checking out process, and the other is the gaps between the arrival times (inter arrival time) of each customer per hour. In order to get an overall perspective of the customer's quality of service, the questionnaires which indicate the result in percentages, are also used to get the evaluation from the customers directly.

There are five counters in the super market, which means consisting of five servers with five queues in terms of Queuing Theory. A queue forms whenever current demand exceeds the existing capacity to serve when each counter is 
so busy that arriving customers cannot receive immediate service facility. So each server process is done as a queuing model in this situation.

The data used in the Queuing model is collected for an arrival time of each customer in two days by the questionnaire form. The observations for number of customers in a queue, their arrival-time and departure-time were taken without distracting the employees. The whole procedure of the service unit each day was observed and recorded using a time-watch during the same time period for each day. In addition, the questionnaires are conducted at the same timings for each day.

\section{Methodology}

Customers requiring service are generated over time by an input source. The required service is then performed for the customers by the service mechanism, after which the customer leaves the queuing system. We can have following two types of models: One model will be as Single-queue Multiple-Servers model (fig.1) and the second one is Multiple-Queues, Multiple-Servers model (fig.2) (Sheu,C., Babbar S. (Jun 1996)).

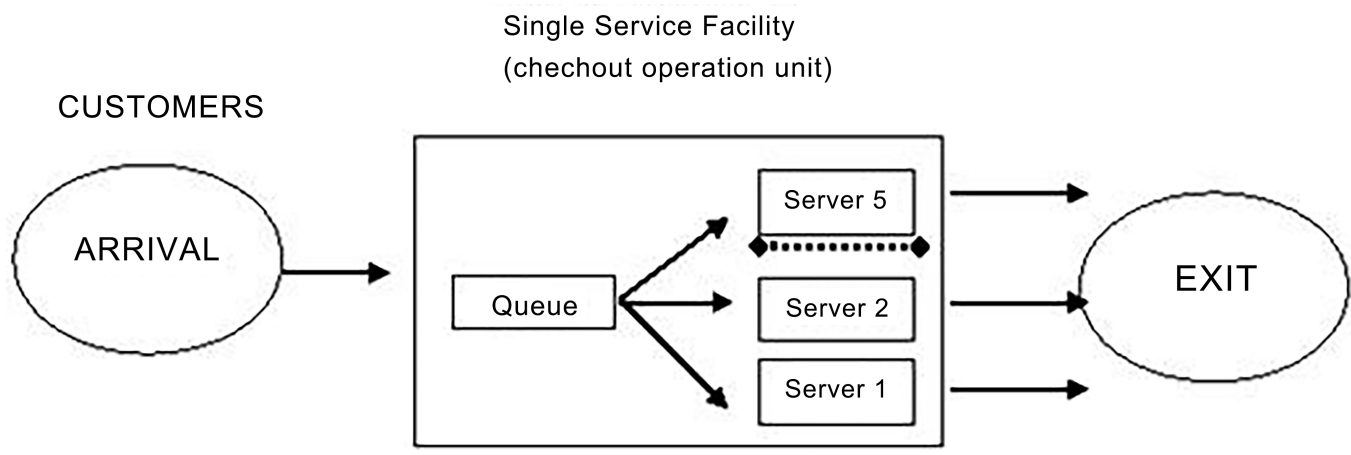

Figure 1. Singer Stage Queuing Model with Single-queue and Multiple Parallel Servers

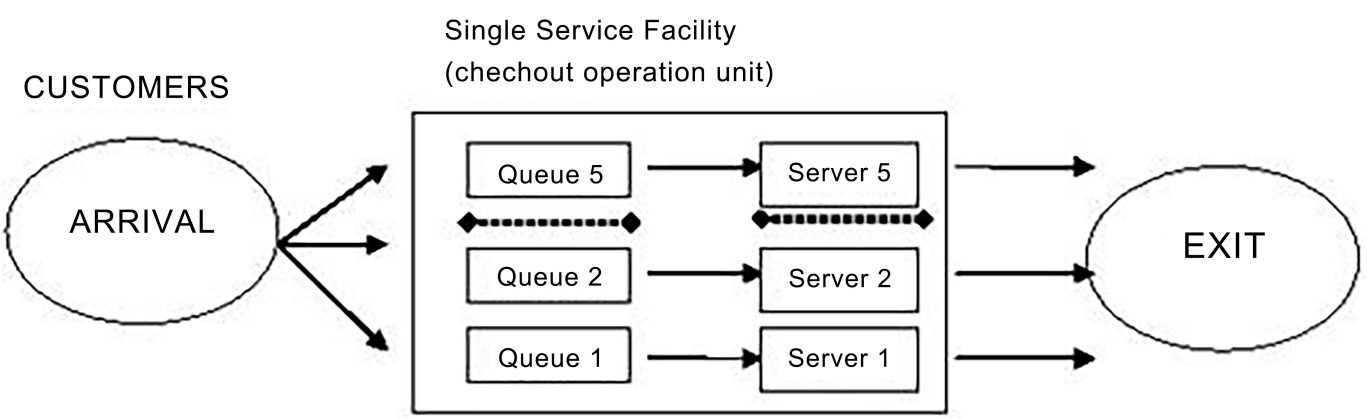

Figure 2. Singer Stage Queuing Model with Multiple Queues and Multiple Parallel Servers 
The supermarket may consist of multiple units to perform same checkout operation of sales, which are usually set all together besides the entrance of the super market. Each unit contains one employee. This kind of a system is called a multiple-server system with single service facility, in other words multiple checkouts counters (service units) with sales checkout as a service available in a system. For such a model the following assumptions are made:

(a) Arrivals of customers follow a Poisson process

(b) Inter arrival times of a Poisson process are exponentially distributed

(c) Service times are exponentially distributed

(d) Identical service facilities (same sales checkout service on each server)

(e) No customer leaves the queue without being served

(f) Infinite number of customers in queuing system of ICA (i.e. no limit for queue capacity)

(g) FIFO (First In First Out) or FCFS (First Come First Serve)

Parameters in Queuing Models (Multiple Servers, Multiple Queues Model)

$n$ =Number of total customers in the system (in queue plus in service)

$C=$ Number of parallel servers (Checkout sales operation units in supermarket)

$\lambda=$ Arrival rate $(1 /$ (average number of customers arriving in each queue in a system in one hour))

$\mu=$ Serving rate $(1 /$ (average number of customers being served at a server per hour))

$C \mu=$ Serving rate when $C>1$ in a system

$\rho=$ System intensity or load, utilization factor $(=\lambda /$ $C \mu$ ) (the expected factor of time the server is busy that is, service capability being utilized on the average arriving customers)

$p_{0}=$ Steady-state Probability of all idle servers in the system

$p_{n}=$ Steady-state Probability of exactly $\mathrm{n}$ customers in the system

$L_{q}=$ Average number of customers in the waiting line (queue)

$W_{q}=$ Average waiting time a customer spends waiting in line excluding the service time

$L_{s}=$ Average number of customers in the system

$W_{s}=$ Average waiting time a customer spends waiting in line including the service time

\section{Results and Discussion}

A sales checkout service has 5 waiting lines in a form of parallel cash counters. Customers are served on a first-come, first-served (FIFO) basis as a salesman of checkout operation unit becomes free. The data has been collected for only two out of five servers on Wednesday (weekday) by using questionnaires. It was assumed that the customers' crowd is more, on average, on weekday. Although the sales checkout unit has 5 parallel counters out of which 2 were observed (each of them has an individual salesman to deal with the customers in a queue), it is possible that some of the checkout units are idle.

We can estimate confidence intervals for average service rate and average arrival rate. Assuming service time and arrival time are iid with $\mathrm{N}(0,1)$, then the $95 \%$ confidence interval for arrival rate can be:

Mean $($ service time $)=01: 06$ minutes per customer $(\mathrm{read}$ clock as min:sec)

$\mathrm{SD}($ service time $)=00: 06 \mathrm{~min}$

Mean (arrival time) $=00: 37$ min per customer

$\mathrm{SD}$ (arrival time) $=00: 06 \mathrm{~min}$

And $n=41$ customers

95\% Confidence Intervals for Service Time:

Mean (service time) $-1.96($ SE $($ service time $))=54$ sec/customer

Mean $($ service time $)+1.96(\mathrm{SE}($ service time $))=78$ sec/customer

95\% Confidence Intervals for Service Rate:

$1 /[$ Mean(service time $)+1.96(\mathrm{SE}($ service time $))]=$ $0.01282=46$ customers $/ \mathrm{sec}$

$1 /[$ Mean(service time $)-1.96(\mathrm{SE}($ service time $))]=$ $0.01852=67$ customers $/ \mathrm{sec}^{* *}$

$* *(0.01852 \mathrm{sec} * 60 * 60)$

95\% Confidence Intervals for Arrival Time:

Mean (arrival time $)-1.96(\mathrm{SE}($ arrival time $))=24 \mathrm{sec}$ /customer

Mean $($ arrival time $)+1.96(\mathrm{SE}($ arrival time $))=49 \mathrm{sec}$ /customer

95\% Confidence Intervals for Arrival Rate:

$1 /[$ Mean(arrival time $)+1.96$ ( $\mathrm{SE}($ arrival time $))]=$ $0.02041=73$ customers $/ \mathrm{sec}^{* *}$

$1 /[$ Mean(arrival time) - 1.96 (SE(arrival time) $)]=$ $0.04167=150$ customers $/ \mathrm{sec}$

$* *(0.02041 \mathrm{sec} * 60 * 60)$

The confidence intervals show that 73 to 150 customers arrive in 2-server system within an hour whereas 46 to 67 customers are served. That means there are still some customers not being served and are waiting for their turn in a queue to be served. This is due to a service time provided by a server to the customers.

\section{Expected Queue Length}

We can find the expected length of queue by using empirical data. In survey, the number of customers waiting in a queue was observed. The average of that number in a system is $(1+1+3+\ldots+2+0) / 41=2.07$ customers per minute on average waiting in a queue in a system within $25 \mathrm{~min}$ of data collection time.

Queuing Analysis 
On Wednesday (weekday), customers arrive at an average of 98 customers per hour, and an average of 55 customers can be served per hour by a salesperson.

$$
\begin{aligned}
& n=41 \\
& C=2 \\
& \lambda=98 \text { customers per hour } \\
& \mu=55 \text { customers per hour } \\
& C \mu=(2)(55)=110 \text { (service rate for } 2 \text { servers ) } \\
& \rho=0.8909 \\
& p_{0}=0.5769 \\
& L_{q}=6.8560 \\
& W_{q}=0.0700 \text { hours }
\end{aligned}
$$

The performance of the sales checkout service on weekday is sufficiently good. We can see that the probability for servers to be busy is 0.8909 , i.e. $89.09 \%$. The average number of customers waiting in a queue is $\mathrm{Lq}$ $=6.8560$ customers per 2 -server. The waiting time in a queue per server is $\mathrm{Wq}=4.2 \mathrm{~min}$ which is normal time in a busy server.

The average queue length can be estimated simply from raw data from questionnaires by using the collected number of customers waiting in a queue each minute. We can compare this average with that of queuing model. The empirical analysis of queuing system of the supermarket is that they may not be very efficient in terms of resources utilization. Queues form and customers wait even though servers may be idle much of the time. The fault is not in the model or underlying assumptions. It is a direct consequence of the variability of the arrival and service processes. If variability could be eliminated, system could be designed economically so that there would be little or no waiting, and hence no need for queuing models.

\section{Conclusions}

With the increasing number of customers coming to the super market for shopping, either for usual grocery or for some house wares, there is a trained employee serving at each service unit. Sales checkout service has sufficient number of employees (servers) which is helpful during the peak hours of weekdays. Other than these hours, there is a possibility of short Queues in a model and hence no need to open all checkouts counters for each hour. Increasing more than sufficient number of servers may not be the solution to increase the efficiency of the service by each service unit.

\section{REFERENCES}

[1] Adan, I.J.B.F., Boxma1, O.J., Resing, J.A.C. (2000), "Queuing models with multiple waiting lines,"

[2] Department of Mathematics and Computer Science, Eindhoven University of Technology,

[3] Banks, J., Carson, J. S., Nelson, B. L., Nicol, D. M. (2001), Discrete-Event System Simulation, Prentice Hall international series, 3rd edition, p24-37

[4] Sheu, C., Babbar S. (Jun 1996), “A managerial assessment of the waiting-time performance for

[5] alternative service process designs, " Omega, Int. J. Mgmt Sci. Vol. 24, No. 6, pp. 689-703 